\title{
What's PrEP?: peer navigator acceptability among minority MSM in Washington
}

\author{
Jade Pagkas-Bather ${ }^{1,2^{*}}$ (D) Jahn Jaramillo ${ }^{3}$, Jsani Henry ${ }^{4}$, Vanessa Grandberry ${ }^{5}$, Luis F. Ramirez ${ }^{6}$, Lorenzo Cervantes ${ }^{7}$, \\ Joanne D. Stekler ${ }^{1}$, Michele P. Andrasik ${ }^{8}$ and Susan M. Graham ${ }^{1}$
}

\begin{abstract}
Background: Peer navigation is a promising strategy to link at-risk minority men who have sex with men (MSM) to HIV prevention services including pre-exposure prophylaxis (PrEP).

Methods: Thirty-two Black and 63 Latinx HIV-negative MSM living in western Washington completed a survey examining attitudes towards peer navigation and PrEP. Factor analysis derived a score for peer navigator acceptability, and linear regression identified associations with this outcome.

Results: Forty-eight percent were interested in peer navigation. Being insured, higher sexual stigma, and higher PHQ-9 score were associated with higher acceptability, while higher income and having a regular medical provider were associated with lower acceptability. In multivariable analysis, higher sexual stigma predicted higher acceptability, while higher income predicted lower acceptability. Men preferred that peers be matched on sexual orientation, race, age and culture.

Conclusion: Peer navigation interventions to reach minority men should address stigma, focus on lower-income men, and try to match peers to clients to the extent possible.
\end{abstract}

Keywords: HIV prevention, Pre-exposure prophylaxis, Black, Latinx, Men who have sex with men

\section{Background}

Pre-exposure prophylaxis (PrEP) using oral emtricitabine and tenofovir in a combination pill reduces the risk of HIV transmission if adherence is high or intermittent dosing is timed to sexual activity $[1,2]$. In the United States, PrEP has become popular among many White men who have sex with men (MSM), but has, to date, failed to gain a firm foothold among Black and Latinx populations, who are disproportionately impacted by HIV [3]. In 2016, 73\% of PrEP users were White, 10\% were Black (12\% in 2015), and $13 \%$ were Latinx [4, 5], similar to the make-up of the general U.S. population [6, 7]. In contrast, White MSM made up only $22 \%$ of new HIV infections, while Black MSM made up 31\% and Latinx MSM made up 22\% of 33,210 new infections in the United States in 2016 [6, 7]. Black and Latinx MSM

\footnotetext{
* Correspondence: jpagkasbather@medicine.bsd.uchicago.edu; jadevpb@gmail.com

'Department of Medicine, University of Washington, Seattle, WA, USA ${ }^{2}$ Department of Medicine, Section of Infectious Diseases \& Global Health, The University of Chicago, 5841 South Maryland Avenue, Chicago, IL 60637, USA Full list of author information is available at the end of the article
}

are disproportionately at risk for HIV compared to White MSM, yet less likely to use PrEP for HIV prevention.

For a number of reasons, Black and Latinx MSM lack equal access to HIV prevention resources. Minority MSM experience discrimination from within and outside their communities, as a result of both race/ethnicity and sexual minority status $[8,9]$. Structural and economic inequities, such as lack of health insurance, lower income, higher rates of unemployment, and incarceration further widen the gap between HIV-related health outcomes in Black and Latinx MSM compared to their White counterparts [10-14].

Peer navigators represent one potential strategy to address disparities in HIV acquisition and PrEP uptake among minority MSM, by offering a layperson's perspective on HIV prevention and helping men link to and navigate the healthcare system. Such assistance may be especially important, given the role of medical mistrust as a barrier to care in the Black and Latinx communities $[15,16]$. PrEP-utilizing peer navigators may help combat

(c) The Author(s). 2020 Open Access This article is distributed under the terms of the Creative Commons Attribution 4.0 International License (http://creativecommons.org/licenses/by/4.0/), which permits unrestricted use, distribution, and reproduction in any medium, provided you give appropriate credit to the original author(s) and the source, provide a link to the Creative Commons license, and indicate if changes were made. The Creative Commons Public Domain Dedication waiver (http://creativecommons.org/publicdomain/zero/1.0/) applies to the data made available in this article, unless otherwise stated. 
medical mistrust and fill in PrEP-related knowledge gaps that exist among minority MSM [17-20]. For example, in a cohort of young Black MSM in Chicago, discussions with confidants regarding HIV prevention were associated with greater $\mathrm{PrEP}$ awareness $(\mathrm{aOR}=2.26,95 \% \mathrm{CI}$ 1.00 to 5.09) [21]. Peer navigator interventions targeting the HIV care cascade demonstrate that peers can improve patient engagement and increase medication adherence [22].

In western Washington, Latinx individuals represent a growing population, with many new immigrants from Latin and South America, as well as Latinx Americans migrating to the area [23]. Resettlement often encourages individuals to find new communities with similar culture and language. This process of migration might also encourage gathering and seeking out community, which could facilitate peer navigation among Latinx MSM. Furthermore, the stresses of immigration and the threat of deportation within Latinx communities might increase the importance of peer navigator outreach, as Latinx individuals could be afraid to engage with medical providers, especially those who are not Latinx [24].

In contrast, Black communities in western Washington are dispersing, as many Black families have moved out of metro King County/Seattle due to increased housing costs and gentrification [25, 26]. This migration to the suburbs could make it more difficult for Black MSM to connect to peer navigators, as few LGBTQ organizations are located outside of King County, which includes downtown Seattle $[25,26]$. While both Black and Latinx MSM are key populations at higher risk for HIV, Black MSM may be at even higher risk for HIV transmission given the potential for greater social and cultural isolation in western Washington. For example, Black populations are smaller than Latinx populations in King $(6.8 \%$ vs $9.7 \%$ ), Pierce $(7.5 \%$ vs $10.9 \%)$, Snohomish (3.5\% vs $10.2 \%$ ), and Thurston (3.4\% vs 9.0\%) counties, where this study took place [7]. In a study of Chicago residents conducted by the University of Chicago, Black respondents were less likely to identify as lesbian, gay, bisexual, transgender, or queer than Latinx respondents $(22 \%$ of Latinx respondents vs $14 \%$ of Black respondents identified as LGBTQ) [27]. If this is true of western Washington, Black individuals might not see themselves reflected in local LGBTQ organizations despite their outreach efforts, and may therefore be less likely to seek support from them.

In 2017, the Washington State Department of Health funded the Persons at High Risk (PAHR) Navigation Services program funding community-based organizations and agencies in the state to increase PrEP access and HIV testing, as well as healthcare engagement for individuals not being reached using previous strategies [28]. The What's PrEP? study aimed to evaluate the acceptability of peer navigation for PrEP use among Black and Latinx men living in Western Washington, and to identify factors associated with higher or lower acceptability of this approach. In addition, the study aimed to identify which peer navigator characteristics were most important to minority MSM. By identifying modifiable factors associated with acceptability, study findings could be used to identify potential challenges peer navigators might face and to inform the design of programs for effective peer navigation.

\section{Methods}

\section{Study population}

What's PrEP? and its Spanish language version, Que es $\operatorname{PrEP}$ ? was a cross-sectional study that surveyed nonLatinx Black and Latinx cis and transgender MSM. Participants were required to be HIV-negative, maleidentified Black or Latinx, English or Spanish-speaking, age 16 or older, sexually active with a male in the past 12 months, and residing in the Snohomish, Thurston, Pierce, or King counties of western Washington. Participants were recruited by flyer distribution at events, Facebook posts, and word of mouth, as well as through local HIV prevention and STD clinics and from local community-based organizations including Entre Hermanos, the Center for Multicultural Health $(\mathrm{CMCH})$, PCAF (Pierce County AIDS Foundation), Project Neon, and Gay City Health Project.

\section{Procedures and data collection}

Participants took a survey online using REDCap or inperson using a printed, self-administered questionnaire (Additional file 1). The survey, which we estimated to take $30-45 \mathrm{~min}$ on a computer and up to $60 \mathrm{~min}$ on a cell phone, collected information on demographics, HIV risk behaviors, alcohol and drug use, depressive symptoms, sexual stigma, healthcare access, PrEP use and delivery preferences, and interest in a peer navigator for the use of PrEP. Gender identity was characterized using a two-step process to assess sex at birth and current gender identity [29]. Participants were reimbursed promptly using a hard-copy Visa card or electronic Tango card worth $\$ 40$. A subset of participants underwent an in-depth interview; results of these interviews are reported elsewhere. Names were collected only in order to provide reimbursement or to invite participants for interviews, and surveys were de-identified.

\section{Measures}

\section{Peer navigator acceptability}

Participants were asked four questions about their willingness to use a peer for appointment reminders, pilltaking reminders, advice about PrEP (e.g., managing side effects, adherence, planning for refills), and discussing 
privacy or other concerns surrounding PrEP use. Responses were rated as $1=$ very harmful/interfering, $2=$ somewhat useless or harmful/interfering, $3=$ neither useful nor useless, $4=$ somewhat useful, or $5=$ very useful.

\section{Peer navigator attributes}

Participants were asked seven questions about how important different attributes of a potential peer were, in terms of matching with respect to race, sexual orientation, age, relationship status, income, culture, and neighborhood. Responses were rated as rated as $1=$ not important, 2 = slightly important, 3 =fairly important, $4=$ important, or 5 = very important.

\section{Sociodemographic factors}

Data were collected on participant age, years of education, and monthly income. Race/ethnicity was defined as non-Latinx Black vs Latinx, with the small number of Black Latinx individuals classified as Latinx. Sexual orientation was defined as gay, straight, bisexual, queer, or other. Gender identity was assigned as cisgender if participants were male at birth and identified as male or non-binary/genderqueer and transgender if participants were female at birth and identified as male.

\section{Mental health}

Sexual stigma was measured using Logie's modified China MSM Stigma Scale, which evaluates perceived stigma and discrimination faced by MSM as pertains to safety, family, and relationships [30]. Depressive symptoms were measured using the Patient Health Questionnaire (PHQ-9), a self-administered set of questions used to identify depressive symptoms in clinical settings [31]. Disordered alcohol use was measured using the Alcohol Use Disorders Identification Test (AUDIT) [32]. Nonalcohol substance use was measured using the Drug Abuse Screening Test 10 (DAST-10) [33].

\section{Health care factors}

A series of questions assessed whether participants had health insurance, saw a regular medical provider, had disclosed their MSM status to their provider, had ever tested for HIV, and the time since their last HIV test, and clinic visit [34].

\section{Sexual behavior and PrEP use}

A series of questions adapted from the 2014 CDC PrEP clinical practice guidelines [35] were asked, to screen for high-risk behaviors in the past 12 months. These included condomless anal sex with an HIV-negative man, ongoing relationship with an HIV-positive male partner, treatment for an STI, use of post-exposure prophylaxis (PEP), use of crystal meth, use of poppers, nonprescription injection drug use, and exchange sex for drugs, money or housing. Additional questions asked about participants' PrEP awareness and use, as well as whether participants were interested in starting or continuing PrEP.

\section{Statistical analysis}

Descriptive statistics were used to characterize the study population overall and separately for non-Latinx Black and Latinx participants. Chi square or Fisher exact tests were used to examine differences between categorical variables, and Wilcoxon rank sum tests were used to examine differences in continuous variables across categories, including race/ethnicity. Confirmatory factor analysis was conducted to confirm that observed responses to questions regarding participants' interest in peer navigation for PrEP underlay an unobserved latent variable for peer navigator acceptability. Results of the factor analyses were used to predict an acceptability score (described in results), which was the primary outcome.

Unadjusted linear regression was used to evaluate the association of race/ethnicity, sexual orientation, and other variables of interest with peer navigator acceptability. A full multivariable model was then constructed, including race/ethnicity and sexual orientation a priori and other potential correlates associated with peer navigator acceptability at $p<0.10$ in unadjusted analysis. A final, limited analysis included the two a priori predictors and variables associated with peer navigator acceptability at $\mathrm{p}<0.10$ in the full multivariable analysis. A Wald test was conducted to obtain overall $p$-values for categorical variables included in multivariable analysis.

Descriptive statistics were used to report the proportion of participants who rated each peer attribute as "important" or "very important," both overall and by race/ ethnicity category. Participants' ratings of the importance of each peer navigator attribute were graphed using a stacked horizontal bar chart, with color coding for each Likert scale response. Responses of non-Latinx Black and Latinx participants were compared using Wilcoxon rank sum tests and presented in a bar graph. The correlations between peer navigator attribute ratings and acceptability were examined using Spearman's correlation coefficient.

\section{Ethical considerations}

Study procedures and survey questions were developed in collaboration with community partners at Entre Hermanos, the Center for Multicultural Health, and the King County Health Department. Feedback from these and other partners including Gay City, was used to improve recruitment materials and survey wording. The study protocol was approved by the University of Washington Human Subjects Division. All participants provided 
written or electronic informed consent. All data was securely stored on a password protected computer, and all paper forms were stored on a locked unit in a locked cabinet. Data was accessed only by the study investigators.

\section{Results}

Overall, 301 REDCap survey attempts were made, of which 137 were not complete, due to ineligibility or failure to continue to the end, and 164 were complete. Of the 164 completed surveys, 95 were confirmed via email, phone, or in person confirmation of a unique participant. All ninety-five participants were cisgender men; no transgender men enrolled despite efforts to recruit in this category. Thirty-two (34\%) of participants were Black and 63 (66\%) were Latinx (4 of the Latinx participants were also Black, Table 1). Overall 69 (73\%) participants identified as gay, 19 (20\%) identified as bisexual, and $5(5 \%)$ identified as queer/other. The median age was 30 (IQR 26-40) years, with a range of 18 to 66 years of age.

\section{Differences by race/ethnicity}

Black participants were more likely to identify as bisexual or straight rather than gay, relative to Latinx participants (Table $1, p<0.001$ ). In addition, Black participants were older (median 42.5 years vs 29 years, $p=0.001$ ) and more likely to be insured ( $91 \%$ vs $67 \%, p=0.01$ ). Latinx participants were more likely to be foreign born than Black participants $(48 \%$ vs. $6 \%, \mathrm{p}<0.001)$. More Latinx participants were currently using PrEP compared to Black participants $(40 \%$ vs. $25 \%, p=0.19)$, and Latinx participants were more like to have used post-exposure prophylaxis than Black participants $(25 \%$ vs. $6 \%, p=$ 0.02). Latinx participants had higher median sexual stigma scores than Black participants (11 vs. 8, $p=$ 0.004), although more Latinx participants had disclosed their sexual orientation to their medical provider $(54 \%$ vs. $41 \%, p=0.01)$. Overall, Latinx participants reported more high-risk behaviors than Black participants (details in Table 1).

\section{PrEP interest and sexual risk}

Figure 1a presents a pie chart showing the proportion of participants who endorsed at least one high-risk behavior and their PrEP status: 42\% were taking PrEP, 30\% were interested in starting PrEP, $18 \%$ were not taking PrEP and needed more information, and $10 \%$ were not taking PrEP and not interested in PrEP. Fig. 1b presents a pie chart showing the proportion of participants who did not endorse at least one high-risk behavior and their PrEP status: none were taking PrEP, but 13\% were interested in starting PrEP, 69\% were not taking PrEP but needed more information, and 19\% were not interested in PrEP. This difference was significant $(p<0.001)$.

\section{Acceptability of a peer navigator}

Overall, $48 \%$ of participants were interested in a peer navigator for PrEP services, $41 \%$ were "maybe" interested, and $11 \%$ were not interested. Exploratory factor analysis confirmed that observed responses to questions on peer navigator acceptability reflected a single, unobserved latent variable or factor with an Eigenvalue of 3.33, associated with higher values of each response [36]. Factors with an Eigenvalue of 1 of greater are to be retained and are considered to be associated with the latent variable in question [37]. Uniqueness values ranged from 0.33 to 0.50 ; therefore, all question responses were retained. Rotated factor loadings were used to predict an index score for peer navigator acceptability for each participant, which was used as the primary outcome for regression analyses.

Race/ethnicity and sexual orientation were not independent predictors of peer navigator acceptability score, in unadjusted or adjusted analysis (Table 2). In the unadjusted model, having health insurance (beta $=0.55$, 95\% CI 0.11 to 0.99 ), higher sexual stigma score (beta $=$ 0.05 , 95\% CI 0.01 to 0.09 ) and higher PHQ-9 score (beta $=0.04,95 \%$ CI 0.01 to 0.07 ) were associated with higher peer navigator acceptability. Monthly income greater than $\$ 3500$, relative to $\$ 0$ to $\$ 1500$ (beta $=-$ $0.80,95 \% \mathrm{CI}-1.26$ to -0.34 ) and having a regular medical provider (beta $=-0.63,95 \%$ CI -1.03 to -0.23 ) were both associated with lower peer navigator acceptability. In the full multivariable model, no variables were associated with peer navigator acceptability. In the limited multivariable model, income greater than $\$ 3500$ was negatively associated while sexual stigma was positively associated with peer navigator acceptability (adjusted beta $=-0.62,95 \% \mathrm{CI}-1.10$ to -0.14 ; and adjusted beta $=0.04,95 \%$ CI, 0 to 0.08 , respectively).

\section{Exploration of associations between sexual stigma and other variables}

Because higher sexual stigma score was associated with higher peer navigator acceptability in these analyses, we used Wilcoxon rank sum tests to examine associations between sexual stigma score and the following dichotomous variables: ever having tested for HIV, report of any highrisk behavior, disclosure of MSM status to medical provider, report of any STD in the past 12 months, and current PrEP use. Having a STD in the past 12 months was the only variable tested that was significantly associated with higher sexual stigma score $(p=0.03$; median stigma score 11.5 and 9 for those with and without an STD in the past 12 months, respectively). In addition, there were positive Spearman correlations between stigma score and both PHQ-9 score $(\rho=0.24, p=0.02)$ and AUDIT score $(\rho=0.28, p=0.01)$. No correlation was found between stigma and DAST scores $(\rho=0, p=0.98)$. 
Table 1 Study population, with comparison of Black and Latinx MSM

\begin{tabular}{llll}
\hline Variable & non-Latinx & Latinx & P-value \\
& Black & $n=63$ & \\
& $n=32$ & $(\mathrm{~N}, \%$ or & \\
& $(\mathrm{N}, \%$ or & median, IQR) \\
& median, IQR) & &
\end{tabular}

\section{Sociodemographic factors}

Sexual orientation

Gay

Straight

Bisexual

Queer

Age (years)

Education

$<$ high school
high school
some college
college
graduate schoo
nsured (yes)
Income
\$0-\$1500
$\$ 1501-\$ 3500$
$>\$ 3500$

Foreign Born (yes)

Current PrEP use and interest

Currently taking PrEP (yes)
Interested but not taking
PrEP

Not interested in taking PrEP

Ever prescribed PrEP (yes)

Ever taken PrEP (yes)

Mental health

Stigma score
PHQ-9 score
DAST score
AUDIT score
Healthcare factors
Regular medical provider
(yes)
Disclosed sexual orientation
to provider (yes)
Sexual health
Ever tested for HIV
Time since last HIV test $\mathrm{t}^{\mathrm{a}}$
$<3$ months

$\begin{array}{lll}8(25) & 25(40) & \\ 8(25) & 18(28) & 0.19 \\ 16(50) & 20(32) & \\ 8(23) & 27(77) & 0.32 \\ 8(21) & 31(79) & 0.02\end{array}$

$8(3-12) \quad 11(8-14) \quad 0.004$

$7(3-10) \quad 7.5(4-12.5) \quad 0.16$

$1(0-6) \quad 1(0-2) \quad 0.13$

$5(3-15) \quad 5.5(4-11) \quad 0.98$

$21(66) \quad 38(60) \quad 0.30$

$13(41) \quad 34(54) \quad 0.01$

$29(91) \quad 58(92)$

0.81

$16(55) \quad 35(60)$

0.82
Table 1 Study population, with comparison of Black and Latinx MSM (Continued)

\begin{tabular}{|c|c|c|c|}
\hline Variable & $\begin{array}{l}\text { non-Latinx } \\
\text { Black } \\
n=32 \\
\text { (N, \% or } \\
\text { median, IQR) }\end{array}$ & $\begin{array}{l}\text { Latinx } \\
n=63 \\
\text { (N, } \% \text { or } \\
\text { median, IQR) }\end{array}$ & $P$-value \\
\hline $3-6$ months & $5(17)$ & $12(21)$ & \\
\hline $6-12$ months & $4(14)$ & $5(9)$ & \\
\hline$>12$ months & $4(14)$ & $6(10)$ & \\
\hline $\begin{array}{l}\text { Time since last clinic visit } \\
\text { (months) }\end{array}$ & $3(1-5)$ & $2(1-3)$ & 0.30 \\
\hline \multicolumn{4}{|l|}{ High-risk behavior } \\
\hline $\begin{array}{l}\text { Condomless anal sex with HIV } \\
\text { negative man }\end{array}$ & $15(47)$ & $50(79)$ & 0.001 \\
\hline $\begin{array}{l}\text { Relationship with HIV } \\
\text { positive partner }\end{array}$ & $1(3)$ & $7(11)$ & 0.19 \\
\hline $\begin{array}{l}\text { STI treatment in last } 12 \\
\text { months }\end{array}$ & $7(22)$ & $36(57)$ & 0.002 \\
\hline Use of PEP in last 12 months & $2(6)$ & $16(25)$ & 0.02 \\
\hline Use of crystal meth & $5(16)$ & $5(8)$ & 0.25 \\
\hline Use of poppers & $8(25)$ & $27(43)$ & 0.09 \\
\hline Non-prescription IVDU & $1(3)$ & $3(5)$ & 0.72 \\
\hline $\begin{array}{l}\text { Exchange sex for drugs, } \\
\text { money, housing }\end{array}$ & $4(13)$ & $3(5)$ & 0.17 \\
\hline $\begin{array}{l}\text { Any report of these } \\
\text { high-risk behaviors }\end{array}$ & $22(69)$ & $57(90)$ & 0.01 \\
\hline \multicolumn{4}{|l|}{ Importance of peer attributes } \\
\hline \multicolumn{4}{|l|}{ Peer attributes ranked as } \\
\hline \multicolumn{4}{|l|}{ important/very important } \\
\hline Same sexual orientation & $23(72)$ & $47(74)$ & 0.56 \\
\hline Same race & $16(50)$ & $34(54)$ & 0.07 \\
\hline Same age & $16(50)$ & $40(63)$ & 0.33 \\
\hline Same culture & $13(41)$ & $28(44)$ & 0.35 \\
\hline Same neighborhood & $9(28)$ & $27(43)$ & 0.17 \\
\hline Same income & $7(22)$ & $21(33)$ & 0.14 \\
\hline
\end{tabular}

a 8 participants did not report the time since their last HIV test

\section{Peer attributes}

Overall, $73 \%$ of participants rated having a peer navigator of the same sexual orientation as "important" or "very important." The proportion of participants who rated sameness of peer attributes as "important" or "very important was $53 \%$ for race, $49 \%$ for age, $43 \%$ for culture, $38 \%$ for relationship status, and $38 \%$ for living in the same neighborhood (Fig. 2). The median rating by Latinx participants for same race, same culture, and same neighborhood were each higher when compared to the median rating by Black participants (Fig. 3), but these differences were not statistically significant. Spearman correlations between peer attribute rating and peer navigator acceptability were significant for several peer attributes: age $(\rho=0.29, p=0.01)$, race $(\rho=0.27, p=$ 


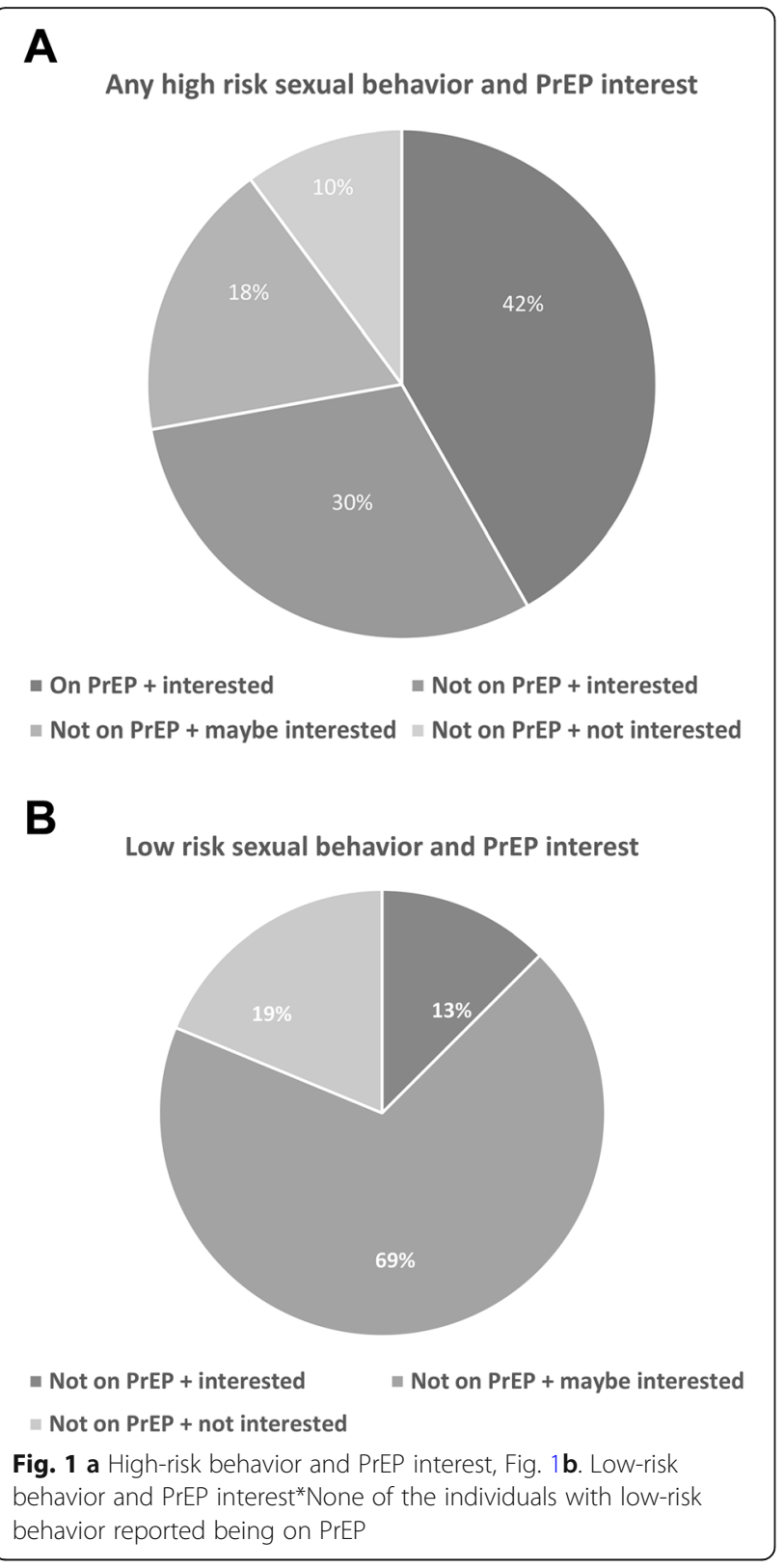

$0.01)$, culture $(\rho=0.35, p=0.0008)$, sexual orientation $(\rho=0.30, p=0.01)$, and neighborhood $(\rho=0.34, p=$ 0.001) (Table 3).

\section{Discussion}

The goal of our study was to gauge acceptability of a peer navigator for PrEP services among Black and Latinx MSM in western Washington. Overall interest in peer navigation was moderate, with just under half of participants expressing interest in PrEP navigation services, suggesting that peer navigation should be one of multiple strategies to engage MSM of color in HIV prevention efforts. Men with insurance and those with higher sexual stigma scores and higher PHQ-9 scores had higher acceptability scores, while men with higher income and regular medical providers had lower acceptability scores. Sexual stigma and income were independent predictors of peer navigator acceptability in multivariable analysis. Of interest, higher sexual stigma was associated with having a sexually transmitted infection (STI) in the past year. The majority of participants preferred a PrEP peer navigator with similar sexual orientation, race/ethnicity, and age as themselves. Overall, there were modest correlations between matching on peer attributes and peer navigator acceptability.

Our study did not require endorsement of sexual activity considered high risk by the CDC as an eligibility requirement, and we did not ask about number of sexual partners. There are multiple studies showing that minority MSM have small sexual networks compared to White MSM, with higher rates of HIV and bacterial STI acquisition despite this smaller size [38-40]. HIV acquisition is often heavily influenced by structural factors such as lack of insurance and access to healthcare, in addition to the usual risk factors for HIV seroconversion such as multiple sex partners, unprotected sexual encounters, and sex under the influence of drugs or alcohol [35, 41-43]. This rationale and a concern that sexual risk behavior is often underreported due to social desirability bias drove our selection of inclusion criteria for this study. In addition, we were interested in evaluating overall acceptance of peer navigation for PrEP among minority MSM as a group, as community norms are often an important factor in determining both stigma related to HIV prevention uptake and outcomes including the uptake of services [44], such as peer navigation for PrEP.

In the HIV Prevention Trials Network (HPTN) 061 study, which investigated strategies for HIV prevention among Black MSM in 6 major US cities, peer navigators were used to promote participant retention [45]. Participants who accepted peer navigation services were younger $(p=0.03)$ and more likely to be retained in the study than those who did not accept peer navigation $(p<0.001)$ [45]. At least one published study has found that gay identity was correlated with less social isolation and more willingness to engage with a peer outreach worker [46]. While sociodemographic factors (age, race/ethnicity, identification as gay) were not strong predictors of peer navigator acceptability among the Black and Latinx MSM in our study, these factors may still be important in other contexts and during actual PrEP delivery via peers.

In What's PrEP?, higher monthly income was associated with lower peer navigator acceptability. Interestingly, the HPTN 073 study of PrEP uptake and adherence among Black MSM found that men with higher incomes had higher rates of PrEP adherence, 
Table 2 Regression analysis of factors associated with peer navigator acceptability

\begin{tabular}{|c|c|c|c|c|c|c|}
\hline Attribute & $\begin{array}{l}\text { Unadjusted } \\
\text { beta }(95 \% \mathrm{Cl})\end{array}$ & $P$ value & $\begin{array}{l}\text { Model } 1 \\
\text { (full multivariable): } \\
\text { Adjusted beta }(95 \% \mathrm{Cl})\end{array}$ & $P$ value & $\begin{array}{l}\text { Model } 2 \\
\text { (limited multivariable): } \\
\text { Adjusted beta }(95 \% \mathrm{Cl})\end{array}$ & $P$ value \\
\hline \multicolumn{7}{|l|}{ Sociodemographic factors } \\
\hline \multicolumn{7}{|l|}{ Race/ethnicity } \\
\hline Non-Latinx Black & Reference & 0.17 & Reference & 0.83 & $0.15(-0.30$ to 0.61$)$ & 0.51 \\
\hline Latinx & $0.30(-0.13$ to 0.72$)$ & & $-0.05(-0.57$ to 0.47$)$ & & & \\
\hline \multicolumn{7}{|l|}{ Sexual orientation } \\
\hline Gay & Reference & & Reference & & Reference & \\
\hline Straight & $-0.98(-2.30$ to 0.34$)$ & 0.15 & $-1.09(-2.72$ to 0.24$)$ & & $-0.77(-2.05$ to 0.51$)$ & 0.32 \\
\hline Bisexual & $0.04(-0.46$ to 0.55$)$ & & $-0.02(-0.58$ to 0.54$)$ & 0.26 & $0.07(-0.45$ to 0.58$)$ & \\
\hline Queer & $0.75(-0.11$ to 1.60$)$ & & $0.51(-0.41$ to 1.44$)$ & & $0.61(-0.28$ to 1.50$)$ & \\
\hline Age (years) & $0.00(-0.02$ to 0.02$)$ & 0.98 & & & & \\
\hline \multicolumn{7}{|l|}{ Education } \\
\hline$<$ High school & Reference & & & & & \\
\hline High school & $0.11(-0.87$ to 1.10$)$ & 0.30 & & & & \\
\hline Some college & $0.07(-0.82$ to 0.96$)$ & & & & & \\
\hline College & $0.04(-0.89$ to 0.97$)$ & & & & & \\
\hline Graduate school & $-0.67(-1.71$ to 0.38$)$ & & & & & \\
\hline Has health insurance & 0.55 (0.11 to 0.99$)$ & 0.02 & $0.27(-0.29$ to 0.82$)$ & 0.34 & & \\
\hline \multicolumn{7}{|l|}{ Monthly income (\$) } \\
\hline$\$ 0-\$ 1500$ & Reference & & Reference & & Reference & \\
\hline$\$ 1501-\$ 3500$ & $0.03(-0.41$ to 0.48$)$ & 0.001 & $0.06(-0.41$ to 0.53$)$ & 0.08 & $0.06(-0.38$ to 0.50$)$ & 0.02 \\
\hline$>\$ 3500$ & $-0.80(-1.26$ to -0.34$)$ & & $-0.54(-1.06$ to -0.02$)$ & & $-0.62(-1.10$ to -0.14$)$ & \\
\hline Foreign-born & $0.34(-0.07$ to 0.76$)$ & 0.10 & & & & \\
\hline \multicolumn{7}{|l|}{ Current PrEP use and interest } \\
\hline Currently taking PrEP (yes) & Reference & & & & & \\
\hline Interested but not taking PrEP & $0.77(0.28$ to 1.26$)$ & 0.005 & & & & \\
\hline Not interested in taking PrEP & $0.08(-0.35$ to 0.52$)$ & & & & & \\
\hline \multicolumn{7}{|l|}{ Mental health } \\
\hline Stigma score & 0.05 (0.01 to 0.09$)$ & 0.006 & $0.03(-0.01$ to 0.07$)$ & 0.14 & 0.04 (0 to 0.08$)$ & 0.03 \\
\hline PHQ-9 score & 0.04 (0.01 to 0.07 ) & 0.01 & $0.02(-0.02$ to 0.05$)$ & 0.36 & & \\
\hline DAST score & $-0.02(-0.10$ to 0.06$)$ & 0.55 & & & & \\
\hline AUDIT score & $0.02(-0.01$ to 0.06$)$ & 0.17 & & & & \\
\hline \multicolumn{7}{|l|}{ Healthcare factors } \\
\hline Has regular medical provider & $-0.63(-1.03$ to -0.23$)$ & 0.002 & $-0.29(-0.76$ to 0.17$)$ & 0.21 & & \\
\hline Disclosed same-sex behavior to provider & $-0.31(-0.91$ to 0.30$)$ & 0.32 & & & & \\
\hline \multicolumn{7}{|l|}{ Sexual health } \\
\hline Ever tested for HIV & $0.37(-0.37$ to 1.10$)$ & 0.32 & & & & \\
\hline \multicolumn{7}{|l|}{ Time since last HIV test } \\
\hline$<3$ months & Reference & & & & & \\
\hline $3-6$ months & $-0.06(-0.57$ to 0.46$)$ & 0.87 & & & & \\
\hline $6-12$ months & $-0.01(-0.70$ to 0.69$)$ & & & & & \\
\hline$>12$ months & $0.25(-0.41$ to 0.91$)$ & & & & & \\
\hline \multicolumn{7}{|l|}{ Time since last clinic visit } \\
\hline$<4$ months & Reference & & & & & \\
\hline
\end{tabular}


Table 2 Regression analysis of factors associated with peer navigator acceptability (Continued)

\begin{tabular}{|c|c|c|c|c|c|c|}
\hline Attribute & $\begin{array}{l}\text { Unadjusted } \\
\text { beta }(95 \% \mathrm{Cl})\end{array}$ & $P$ value & $\begin{array}{l}\text { Model } 1 \\
\text { (full multivariable): } \\
\text { Adjusted beta (95\% Cl) }\end{array}$ & $P$ value & $\begin{array}{l}\text { Model } 2 \\
\text { (limited multivariable): } \\
\text { Adjusted beta (95\% Cl) }\end{array}$ & $P$ value \\
\hline 4-12 months & $-0.11(-0.61$ to 0.39$)$ & 0.89 & & & & \\
\hline$>12$ months & $0.05(-0.71$ to 0.81$)$ & & & & & \\
\hline $\begin{array}{l}\text { Reported condomless anal sex with } \\
\text { a man in past } 12 \text { months }\end{array}$ & $0.34(-0.08$ to 0.76$)$ & 0.11 & & & & \\
\hline $\begin{array}{l}\text { Currently in relationship with } \\
\text { HIV-positive partner }\end{array}$ & $0.20(-0.54$ to 0.94$)$ & 0.59 & & & & \\
\hline $\begin{array}{l}\text { Received STI treatment in past } \\
12 \text { months }\end{array}$ & $0.27(-0.13$ to 0.67$)$ & 0.19 & & & & \\
\hline Use of PEP in past 12 months & $0.10(-0.40$ to 0.59$)$ & 0.70 & & & & \\
\hline Any high-risk behavior & $0.45(-0.09$ to 0.99$)$ & 0.10 & & & & \\
\hline Use of crystal meth in past 12 months & $0.51(-0.14$ to 1.20$)$ & 0.12 & & & & \\
\hline Use of poppers in past 12 months & $-0.20(-0.61$ to 0.21$)$ & 0.34 & & & & \\
\hline Non-prescription IVDU in past 12 months & $0.54(-0.42$ to 1.50$)$ & 0.27 & & & & \\
\hline $\begin{array}{l}\text { Exchange sex for drugs, money, housing } \\
\text { in past } 12 \text { months }\end{array}$ & $0.45(-0.34$ to 1.24$)$ & 0.26 & & & & \\
\hline
\end{tabular}

*Missing some data for last HIV test

suggesting that men with means may need limited or no help engaging with HIV prevention services [47]. In our study, having a regular medical provider was associated with lower peer navigator acceptability, although this factor was not significant in multivariable analysis. In a study by Santelli et al. among adolescents in the United States, confidential visits with a medical provider were associated with discussions about sensitive topics, including sexual orientation and HIV, suggesting that having a trusted regular provider increases access to HIV prevention services [48]. Similarly, although the association was not significant, peer navigator acceptability in our study was lower among participants who had disclosed their sexual orientation to a regular provider. Men who are "out" to their providers may feel that they do not need peer navigation services; conversely, a peer navigator may play an important supporting role when a medical provider is not LGBTQ-friendly. Finally, we found a positive association between having insurance and peer navigator acceptability, although this factor was not significant in multivariable analysis. One hypothesis to explain this finding is that individuals with insurance have fewer concerns about costs, and are therefore more willing to explore novel interventions for HIV prevention. In addition, having insurance is positively correlated with access to preventative health services, including PrEP [49].

Mental health has been identified as a barrier to HIV care engagement among young MSM, and peer navigation has been proposed to address this problem [50]. An

\section{Importance of peer attributes}

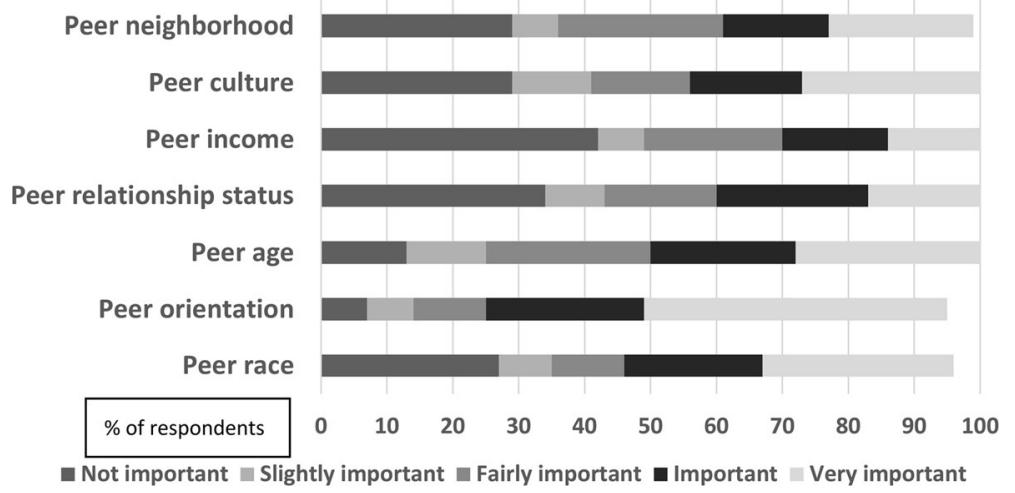

Fig. 2 Likert scale responses for each peer attribute 


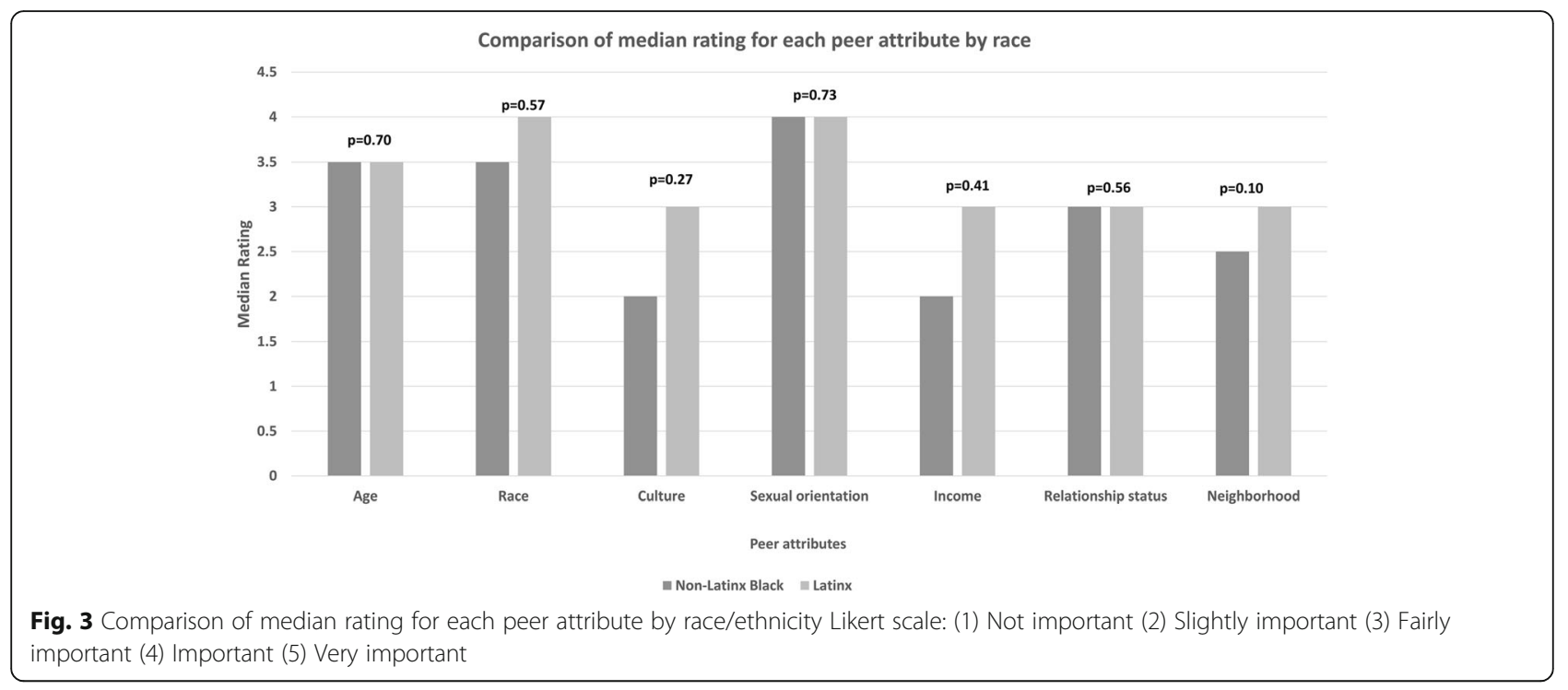

ethnographic study of Black MSM in New York cited internalized homophobia, stigma, and family rejection as barriers to PrEP use, suggesting that men facing these issues might benefit from greater social support surrounding HIV prevention [51]. In the What's PrEP? study, men with higher sexual stigma and depressive symptom scores were more accepting of a peer navigator, suggesting that minority MSM with greater emotional and psychiatric needs may be more open to PrEP peer navigation. Potentially, primary care providers could consider referring MSM with higher PHQ-9 scores for peer navigator services. However, depression was not a significant predictor of peer navigator acceptability in adjusted analysis, while sexual stigma, which is not usually assessed in clinical practice, was. Higher sexual stigma scores were also associated with an STI diagnosis in the past 12 months $(p=0.03)$. A recent STI diagnosis may present an important opportunity to reach out to at-risk MSM and offer peer navigation.

When asked about specific peer attributes, almost three quarters of participants ranked having a peer of the same sexual orientation as important or very

Table 3 Correlation of peer attribute rating with peer navigator acceptability

\begin{tabular}{lll}
\hline Characteristic & Spearman correlation $(\rho)$ & $P$-value \\
\hline Same age & 0.29 & 0.01 \\
Same race & 0.27 & 0.01 \\
Same culture & 0.35 & 0.0008 \\
Same orientation & 0.30 & 0.01 \\
Same income & 0.20 & 0.08 \\
Same relationship status & 0.20 & 0.07 \\
Same neighborhood & 0.34 & 0.001 \\
\hline
\end{tabular}

important. Race, age, and culture were also ranked as important or very important by the majority of participants. Based on these findings, peer navigator programs to support PrEP use among MSM of color may need to match peers to clients on sexual orientation, race/ethnicity, and age group in order for programs to be effective. In our analysis, gender-queer or non-conforming participants were more accepting of peer navigator services for PrEP than gay or bisexual identified individuals, and straight-identifying individuals were less accepting, though these differences were not statistically significant. In prior studies involving peer navigation for homeless Black individuals, respondents indicated that having a peer with a similar lived experience was important to them [52]. Same-gender-loving individuals may feel more affirmed with a peer navigator of the same sexual orientation. There were modest positive correlations between specific peer attributes and peer navigator acceptability, with culture and neighborhood being the strongest. Matching on peer navigator attributes when possible may be helpful for programs using peer navigation to support PrEP uptake and adherence among minority MSM.

PrEP marketing that focuses on gay-identifying MSM may be stigmatizing to men in communities of color, especially those who do not identify as gay [53]. For such men, it may be important to emphasize PrEP's value for sexual health and well-being, as opposed to focusing on risk $[53,54]$. Our findings suggest that peer navigation for PrEP among Black and Latinx MSM may be more acceptable to men if they share a similar sexual orientation with the navigator - in many cases, this orientation may be bisexual or fluid. One potential strategy would be to triage minority MSM with a recent STI diagnosis for peer navigation matched on sexual orientation for 
discussions around STI prevention and information about PrEP.

Although the evidence base is still limited, several ongoing studies will examine the role of peer leaders or change agents in efforts to increase PrEP knowledge and use among MSM of color [55, 56]. In a cluster- randomized trial led by Patel et al. in New York City, an intervention focusing on "empowering with PrEP" (E-PrEP) using PrEP-focused social media messaging disseminated by a peer leader will be compared to an E-Health online control in which participants receive no PrEP-specific or HIV-related information [55]. In PrEP Chicago, the effectiveness of peer change agents recruited through social media networks and snowball sampling is being evaluated in an individual-level randomized trial evaluating outcomes including referrals to a PrEP informational hotline; PrEP knowledge, attitudes, and intention for use; and PrEP uptake among study participants [56]. Additional studies are needed to evaluate peer approaches in other settings, especially in areas such as western Washington in which minority communities are more dispersed and face significant barriers to accessing culturally tailored care. These studies should consider mechanisms through which peer support is thought to work, including the reduction of sexual stigma and the provision of social support from those of a similar background.

This study had several limitations. First, power was limited due to small sample size, particularly for Black participants, who only comprised about one third of our sample. In addition, we were unable to enroll any Black or Latinx transgender men. Although the What's PrEP? study team included members of racial and sexual minorities and sought input and collaboration from community-based organizations serving MSM of color in the study area, effectively engaging this population was challenging for cisgender men and unsuccessful for transgender men of color. Mistrust of research may have been a factor, and specifically targeted research may be needed to reach vulnerable transgender men of color. This limited power decreased our ability to identify independent predictors of peer navigator acceptability in multivariable analysis. Second, the study took place in western Washington and our participants' experiences may not be representative of those of MSM of color living in other parts of the United States. Third, online surveys are prone to fraudulent activity, and it is possible that there may have been duplicate surveys completed by the same individual using different contact information, despite the safeguards put in place. In addition, three survey entries for which contact details were not provided could not be confirmed as unique participants, and therefore were excluded. This may have resulted in underestimation or overestimation of peer navigator acceptability. Fourth, despite recruitment of both cis and transgender MSM using a two-step gender identity process, only cisgender MSM enrolled in our study. Perhaps partnering with a transgender-specific communitybased organization would have helped to increase the participation of transgender MSM. Finally, because peer navigator acceptability in this study was measured in the abstract, actual acceptability remains unclear and may be higher or lower.

\section{Conclusion}

Men of color who engage in HIV prevention efforts using PrEP may benefit from a peer navigator matched to their sexual orientation, race/ethnicity, age, and potentially other attributes. Men who may require additional emotional support or trauma-informed care due to stigma and discrimination, factors making them more vulnerable to HIV, may be more likely to seek out peer navigation in efforts to prevent HIV. MSM who had higher incomes were less accepting of a peer navigator, suggesting that offering peer navigation services to men with more means and greater access to care should not be a high priority. Clinics or organizations wishing to implement PrEP peer navigation programs for Black and Latinx MSM may want to consider the utility of matching peers to participants by sexual orientation, race/ethnicity, and age, as well as screening for sexual stigma and depression. Psychologically vulnerable MSM may have a greater need for a peer navigator, who may need to help men link to mental health services in addition to HIV prevention services. Furthermore, men with recent STI diagnoses and high sexual stigma scores may be appropriate targets for peer navigation to prevent HIV and other STI. Our results suggest that peer navigation is promising and should be incorporated into a suite of options for engagement and prevention of HIV among minority MSM, if it proves to be cost effective in future studies.

\section{Supplementary information}

Supplementary information accompanies this paper at https://doi.org/10. 1186/s12889-020-8325-5.

Additional file 1. What's PrEP Study Questionnaire. Questions and response choices used in the study.

\section{Abbreviations \\ Cl: Confidence interval; E-PrEP: Empowering pre-exposure prophylaxis; HIV: Human immunogenicity virus; HPTN: HIV prevention trials network; LGBTQ: Lesbian, gay, bisexual, transgender, queer; MSM: Men who have sex with men; PCAF: Pierce County AIDS Foundation; PHQ-9: Patient health questionnaire 9; PrEP: Pre-exposure prophylaxis; STD: Sexually transmitted disease; STI: Sexually transmitted infection}

\section{Acknowledgements}

We thank the men who participated in the What's PrEP? and Ques es PrEP? surveys. We are especially grateful to our community partners, who helped 
us recruit participants and invited us into their spaces to learn about the Black and Latinx communities in western Washington. We would like to especially acknowledge Lesster Munguia and Joel Aguirre at Entre Hermanos and Tristan Gardner, formerly at the Center for MultiCultural Health. The following individuals provided input and support for this project: Stephaun Wallace, Kimiam Waters, Andrew Secor, Jane Simoni, Murugi Micheni, and Matthew Golden.

\section{Authors' contributions}

JPB analyzed, interpreted, and wrote the manuscript for the What's PrEP? study. JJ edited the manuscript and provided extensive feedback. JH provided input on the survey questionnaire administered to participants. VG, LFR, and LC helped with recruitment of participants for the study. JDS and MPA edited and provided feedback on the manuscript. SMG was the principal investigator for the project, secured funding for it, and extensively edited the manuscript. All authors read and approved the final manuscript.

\section{Funding}

This research was funded by a 2016 Global to Local developmental grant from the University of Washington / Fred Hutch Center for AIDS Research, an $\mathrm{NIH}$ funded program under award number Al027757 which is supported by the following NIH Institutes and Centers: NIAID, NCI, NIMH, NIDA, NICHD, NHLBI, NIA, NIGMS, NIDDK. SMG was also supported by the Robert W. Anderson Professorship in Medicine. JDS was supported by Washington State Department of Health. The funding bodies had no role in study design, data collection and interpretation, or analysis of the data.

\section{Availability of data and materials}

The datasets analyzed during the current study are available in a REDCap database. They are not available publicly available due to the potential for individual privacy to be compromised, but a de-identified, limited dataset will be shared upon request to the corresponding author.

\section{Ethics approval and consent to participate}

Study procedures and survey questions were developed in collaboration with community partners at Entre Hermanos, the Center for Multicultural Health, and the King County Health Department. Feedback from these and other partners including Gay City, was used to improve recruitment materials and survey wording. The study protocol was approved and overseen by the University of Washington Human Subjects Division (HSD, STUDY00000239). All participants provided either on-line, electronic documentation of consent or written documentation of informed consent, following procedures approved by the ethical review board. All data were encrypted and securely stored on password-protected computers. Data were accessed only by the study investigators.

\section{Consent for publication}

Not applicable.

\section{Competing interests}

There are no financial or non-financial competing interests.

\section{Author details}

'Department of Medicine, University of Washington, Seattle, WA, USA. ${ }^{2}$ Department of Medicine, Section of Infectious Diseases \& Global Health, The University of Chicago, 5841 South Maryland Avenue, Chicago, IL 60637, USA. ${ }^{3}$ Centers for Disease Control and Prevention, Guatemala City, Guatemala. ${ }^{4}$ Seattle \& King County Public Health HIV/STD Program, Seattle, WA, USA. ${ }^{5}$ Center for MultiCultural Health, Seattle, WA, USA. ${ }^{6}$ Entre Hermanos, Seattle, WA, USA. ${ }^{7}$ Pierce County AIDS Foundation, Tacoma, WA, USA. ${ }^{8}$ Fred Hutchinson Vaccine and Infectious Disease Division, Seattle, USA.

\section{Received: 22 August 2019 Accepted: 5 February 2020}

Published online: 18 February 2020

\section{References}

1. Baeten $J M$, Heffron $R$, Kidoguchi $L$, et al. Integrated delivery of antiretroviral treatment and pre-exposure prophylaxis to HIV-1-serodiscordant couples: a prospective implementation study in Kenya and Uganda. PLoS Med. 2016; 13(8):e1002099.
2. Molina JM, Capitant C, Spire B, et al. On-demand Preexposure prophylaxis in men at high risk for HIV-1 infection. NEJM. 2015;373(23):2237-46.

3. Smith DK, Van Handel M, Grey J. Estimates of adults with indications for HIV Pre-Exposure Prophylaxis by jurisdiction, transmission risk group, and race/ ethnicity, United States, 2015. Ann Epidemiol. 2018; 28(12):850-857.e9.

4. Mera M, Hawkins T, Bush S, Rawlings K, McCallister S. (2017). Changes in Truvada for HIV pre-exposure prophylaxis utilization in the USA: 20122016_disparities. Poster session presented at IAS, Paris, France.

5. Highleyman L. PrEP use is rising fast in US, but large racial disparities remain. 2016. http://www.aidsmap.com/PrEP-use-is-rising-fast-in-US-butlarge-racial-disparities-remain/page/3065545/. Accessed 17 July 2019.

6. CDC. Diagnoses of HIV infection in the United States and dependent areas, 2015. 2015. https://www.cdc.gov/hiv/pdf/library/reports/surveillance/cdc-hivsurveillance-report-2015-vol-27.pdf. Accessed 15 July 2018.

7. United States Census Bureau Quick Facts. 2018. https://www.census.gov/ quickfacts/fact/table/seattlecitywashington,US/PST045218. Accessed 15 July 2018.

8. Wohl AR, Galvan FH, Carlos JA, et al. A comparison of MSM stigma, HIV stigma and depression in HIV-positive Latino and African American men who have sex with men (MSM). AIDS Behav. 2013;17(4):1454-64.

9. Arnold EA, Rebchook GM, Kegeles SM. 'Triply cursed': racism, homophobia and HIV-related stigma are barriers to regular HIV testing, treatment adherence and disclosure among young black gay men. Cult Health Sex. 2014;16(6):710-22.

10. Millett, G. (2018). Racial disparities in HIV/STI in the United States [PowerPoint slides]. Retrieved from PDF.

11. CDC National Center for HIV/AIDS, Viral Hepatitis, STD, and TB Prevention Division of HIV/AIDS Prevention. 2017. HIV surveillance by race/ethnicity [PowerPoint slides]. Retrieved from https://www.cdc.gov/hiv/pdf/library/ slidesets/cdc-hiv-surveillance-race-ethnicity-2017.pdf

12. Ransome Y, Kawachi I, Braunstein S, Nash D. Structural inequalities drive late HIV diagnosis: the role of black racial concentration, income inequality, socioeconomic deprivation, and HIV testing. Health Place. 2016;42:148-58.

13. Wildeman C, Wang EA. Mass incarceration, public health, and widening inequality in the USA. Lancet. 2017;389(10077):1464-74.

14. Dumont DM, Allen SA, Brockmann BW, Alexander NE, Rich JD. Incarceration, community health, and racial disparities. J Health Care Poor Underserved. 2013;24(1):78-88.

15. Galvan FH, Bogart LM, Klein DJ, Wagner GJ, Chen YT. Medical mistrust as a key mediator in the association between perceived discrimination and adherence to antiretroviral therapy among HIV-positive Latino men. J Behav Med. 2017:40(5):784-93.

16. Pellowski JA, Price DM, Allen AM, Eaton LA, Kalichman SC. The differences between medical trust and mistrust and their respective influences on medication beliefs and ART adherence among African-Americans living with HIV. Psychol Health. 2017;32(9):1127-39.

17. Grov C, Whitfield TH, Rendina HJ, Ventuneac A, Parsons JT. Willingness to take PrEP and potential for risk compensation among highly sexually active gay and bisexual men. AIDS Behav. 2015;19(12):2234-44.

18. Underhill K, Morrow KM, Colleran C, et al. Explaining the efficacy of preexposure prophylaxis (PrEP) for HIV prevention: a qualitative study of message framing and messaging preferences among US men who have sex with men. AIDS Behav. 2016;20(7):1514-26.

19. Parsons JT, Rendina $H J$, Whitfield $T H$, Grov C. Familiarity with and preferences for oral and long-acting injectable HIV pre-exposure prophylaxis (PrEP) in a national sample of gay and bisexual men in the U.S. AIDS Behav. 2016;20(7):1390-9.

20. Eaton LA, Driffin DD, Bauermeister J, Smith $\mathrm{H}$, Conway-Washington C. Minimal awareness and stalled uptake of pre-exposure prophylaxis (PrEP) among at risk, HIV-negative, black men who have sex with men. AIDS Patient Care STDs. 2015;29(8):423-9.

21. Chen YT, Kolak M, Duncan DT, et al. Neighbourhoods, networks and preexposure prophylaxis awareness: a multilevel analysis of a sample of young black men who have sex with men. Sex Transm Infect. 2019;95(3):228-35.

22. Beach LB, Greene GJ, Lindeman P, et al. Barriers and facilitators to seeking HIV services in Chicago among young men who have sex with men: perspectives of HIV service providers. AIDS Patient Care STDs. 2018;32(11):468-76.

23. Brunner J. Census: Hispanics are state's fastest-growing group. 2011. The Seattle Times. https://www.seattletimes.com/seattle-news/census-hispanicsare-states-fastest-growing-group/. Accessed 29 October 2018.

24. Philbin MM, Flake M, Hatzenbuehler ML, Hirsch JS. State-level immigration and immigrant-focused policies as drivers of Latino health disparities in the United States. Soc Sci Med. 2018;199:29-38. 
25. Scruggs G. Reuters. As Seattle gentrifies, blacks no longer feel welcome. 2018. https://www.reuters.com/article/us-usa-seattle-gentrification/asseattle-gentrifies-blacks-no-longer-feel-welcome-idUSKBN1 KCOQZ. Accessed 29 October 2018.

26. Beason T. Seattle's vanishing black community. The Seattle Times 2016. https://www.seattletimes.com/pacific-nw-magazine/seattles-vanishing-blackcommunity/. Accessed 4 March 2018.

27. Cohen CJ. Millennial attitudes on LGBT issues: Race, identity, and experience. 2018. [PDF file]. Retrieved from http://genforwardsurvey.com/ assets/uploads/2017/05/GenForward-June-2018-LGBT-Report_FINAL.pdf

28. Statewide Prevention Navigation Agencies. 2018. [PDF file]. Retrieved from https://www.doh.wa.gov/Portals/1/Documents/Pubs/150-083PrEPNavigatorsWA.pdf.

29. Institute of Medicine Board on the Health of Select Populations. 2013.The national academies collection: reports funded by National Institutes of Health in collecting sexual orientation and gender identity data in electronic health records: worshop summary. National Academies Press. National Academy of Sciences, Washington, DC.

30. Logie $\mathrm{CH}$, Newman PA, Chakrapani V, Shunmugam M. Adapting the minority stress model: associations between gender non-conformity stigma, HIV-related stigma and depression among men who have sex with men in South India. Soc Sci Med. 2012;74(8):1261-8.

31. Kroenke K, Spitzer RL, Williams JB. The PHQ-9: validity of a brief depression severity measure. J Gen Intern Med. 2001;16(9):606-13.

32. Babor TF, Higgins-Biddle, John C, Saunders, John B, Monteiro, Maristela G \& World Health Organization. Dept. of Mental Health and Substance Dependence. AUDIT : the Alcohol Use Disorders Identification Test : guidelines for use in primary health care Geneva, Switzerland: World Health Organization. 2001. [PDF file]. Retrieved from https://apps.who.int/iris/ bitstream/handle/10665/67205/WHO_MSD_MSB_01.6a.pdf;jsessionid= C8EAFA37CDBC6377832EBB6E04FCEB41? sequence=1.

33. Skinner HA. The drug abuse screening test. Addict Behav. 1982;7(4):363-71.

34. DiNenno EAPJ, Irwin K, et al. Recommendations for HIV screening of gay, bisexual, and other men who have sex with men — United States, 2017. MMWR. 2017;66:830-2.

35. 2014 CDC PrEP clinic practice guidelines. 2014. [PDF file]. Retrieved from https://www.cdc.gov/hiv/pdf/guidelines/PrEPguidelines2014.pdf.

36. Sakaluk JK, Short SD. A methodological review of exploratory factor analysis in sexuality research: used practices, best practices, and data analysis resources. J Sex Res. 2017:54(1):1-9.

37. Gaskin CJ, Happell B. On exploratory factor analysis: a review of recent evidence, an assessment of current practice, and recommendations for future use. Int J Nurs Stud. 2014:51(3):511-21.

38. Newcomb ME, Ryan DT, Garofalo R, Mustanski B. Race-based sexual stereotypes and their effects on sexual risk behavior in racially diverse young men who have sex with men. Arch Sex Behav. 2015;44(7):1959-68.

39. Newcomb ME, Mustanski B. Racial differences in same-race partnering and the effects of sexual partnership characteristics on HIV risk in MSM: a prospective sexual diary study. J Acquir Immune Defic Syndr. 2013; 62(3):329-33.

40. Raymond HF, McFarland W. Racial mixing and HIV risk among men who have sex with men. AIDS Behav. 2009;13(4):630-7.

41. Millett GA, Flores SA, Peterson JL, Bakeman R. Explaining disparities in HIV infection among black and white men who have sex with men: a metaanalysis of HIV risk behaviors. AIDS. 2007:21(15):2083-91.

42. Maulsby C, Millett G, Lindsey K, et al. A systematic review of HIV interventions for black men who have sex with men (MSM). BMC Public Health. 2013;13:625.

43. Murray A, Gaul Z, Sutton MY, Nanin J. "We hide...": Perceptions of HIV risk among Black and Latino MSM in New York City. Am J Mens Health. 2018;12(2):180-8.

44. Stangl AL, Earnshaw VA, Logie $\mathrm{CH}$, et al. The health stigma and discrimination framework: a global, crosscutting framework to inform research, intervention development, and policy on health-related stigmas. BMC Med. 2019;17(1):31.

45. Wheeler DP, Koblin B, Mayer K, et al. Utilizing peer health navigation for retention in an HIV prevention trial [Abstract]. Retrieved from https://www. hptn.org/sites/default/files/inline-files/44_ Utilizing\%20Peer\%20Health\%20Navigation.pdf. Accessed 4 March 2019.

46. Reed SJ, Miller RL. Thriving and adapting: resilience, sense of community, and syndemics among young black gay and bisexual men. Am J Community Psychol. 2016;57(1-2):129-43.
47. Wheeler DP, Fields SD, Beauchamp G, et al. Pre-exposure prophylaxis initiation and adherence among black men who have sex with men (MSM) in three US cities: results from the HPTN 073 study. J Int AIDS Soc. 2019;22(2):e25223.

48. Santelli JS, Klein JD, Song $X$, et al. Discussion of potentially sensitive topics with young people. Pediatrics. 2019;143(2). pii: e20181403.

49. Mikati T, Maloney P, Tabidze I, Mehta SD. The change in insurance status among patients seeking care at Chicago sexually transmitted disease clinics after affordable care act implementation. Sex Transm Dis. 2016:43(4):260-3.

50. Remien RH, Bauman LJ, Mantell JE, et al. Barriers and facilitators to engagement of vulnerable populations in HIV primary care in New York City. J Acquir Immune Defic Syndr. 2015;69(Suppl 1):S16-24.

51. Garcia J, Parker C, Parker RG, Wilson PA, Philbin M, Hirsch JS. Psychosocial implications of homophobia and HIV stigma in social support networks: insights for high-impact HIV prevention among black men who have sex with men. Health Educ Behav. 2016;43(2):217-25.

52. Corrigan PW, Pickett S, Schmidt A, et al. Peer navigators to promote engagement of homeless African Americans with serious mental illness in primary care. Psychiatry Res. 2017;255:101-3.

53. Rivet Amico K, Bekker LG. Global PrEP roll-out: recommendations for programmatic success. Lancet HIV. 2019;6(2):e137-40.

54. Golub SA, Gamarel KE. The impact of anticipated HIV stigma on delays in HIV testing behaviors: findings from a community-based sample of men who have sex with men and transgender women in New York City. AIDS Patient Care STDs. 2013;27(11):621-7.

55. Patel W, Ginsburg Z, Golub SA, et al. Empowering with PrEP (E-PrEP), a peer-led social media-based intervention to facilitate HIV Preexposure prophylaxis adoption among young black and Latinx gay and bisexual men: protocol for a cluster randomized controlled trial. JMIR Res Protoc. 2018;7(8):e11375.

56. Young LE, Schumm P, Alon L, et al. PrEP Chicago: a randomized controlled peer change agent intervention to promote the adoption of pre-exposure prophylaxis for HIV prevention among young black men who have sex with men. Clin Trials. 2018;15(1):44-52.

\section{Publisher's Note}

Springer Nature remains neutral with regard to jurisdictional claims in published maps and institutional affiliations.

\section{Ready to submit your research? Choose BMC and benefit from:}

- fast, convenient online submission

- thorough peer review by experienced researchers in your field

- rapid publication on acceptance

- support for research data, including large and complex data types

- gold Open Access which fosters wider collaboration and increased citations

- maximum visibility for your research: over $100 \mathrm{M}$ website views per year

At $\mathrm{BMC}$, research is always in progress.

Learn more biomedcentral.com/submission 